\title{
Asymmetric light transmission effect based on an evolutionary optimized semi-Dirac cone dispersion photonic structure
}

\author{
Emre Bor, ${ }^{1,2}$ Mirbek Turduev, ${ }^{2, *}$ Utku Görkem Yasa, ${ }^{1,3}$ Hamza Kurt, ${ }^{1}$ and Kestutis Staliunas ${ }^{4,5}$ \\ ${ }^{1}$ Department of Electrical and Electronics Engineering, TOBB University of Economics and Technology, Ankara 06560, Turkey \\ ${ }^{2}$ Department of Electrical and Electronics Engineering, TED University, Ankara 06420, Turkey \\ ${ }^{3}$ TÜBITAK BILLEM / ILTAREN Research Institute, Şehit Yzb. IIlhan Tan Klşlası, Ümitköy, Ankara 06800, Turkey \\ ${ }^{4}$ Departament de Física, Universitat Politècnica de Catalunya, Colom 11, E-08222 Terrassa, Barcelona, Spain \\ ${ }^{5}$ Institució Catalana de Recerca i Estudis Avançats, Passeig Lluís Companys 23, E-08010 Barcelona, Spain
}

(Received 10 October 2018; revised manuscript received 18 November 2018; published 10 December 2018)

\begin{abstract}
In this paper, we present the numerical and experimental demonstration of asymmetric light transmission by a compact photonic crystal (PC) structure, which sustains semi-Dirac cone dispersion. The semi-Dirac point is obtained by exploiting plane-wave expansion method in a rectangular unit cell of a cylindrical dielectric rod, forming a PC structure. The preselected part of the corresponding PC structure is optimized via differential evolution to maximize the transmission efficiency in one direction, and to minimize in the opposite direction. In this regard, finite-difference time-domain method is integrated with the optimization algorithm to numerically design a compact PC structure with asymmetric light transmission effect. The detailed numerical investigation of the optimized PC structure is represented, and the experiments in the microwave regime are performed to verify the numerical results. The experimentally measured transmission efficiency reaches $78 \%$ for one direction, whereas in the opposite direction it reduces to $11 \%$. The physical mechanism of asymmetric light transmission is related to differences of the projection of the wave incident from both directions into the eigenmodes of the photonic structure.
\end{abstract}

DOI: 10.1103/PhysRevB.98.245112

\section{INTRODUCTION}

Over recent years, asymmetric light transmission (ALT) through an optical system has been an attractive research area due to its fundamental role in designing the next generation of all-optical computing systems [1-4]. An optical device that provides large difference in transmittance between the forward and backward light wave can be considered as an ALT device. In general, a straightforward way to obtain ALT is the use of optical nonreciprocity. However, according to the Lorentz reciprocity theorem, regardless of any physical constraints and parameters, the time-reversal symmetry is always preserved for light transport in a linear and timeinvariant optical medium. This means that to break timereversal symmetry the optical system should be operating in a nonlinear regime [5-9] or magneto-optical media must be used [10,11]. However, this method of achieving ALT has several challenging and practical issues such as noncompatibility with current complementary metal-oxide-semiconductor fabrication technology in terms of miniaturization process; magneto-optical inducing could affect the functionalities of other devices in the system, requiring high input power and a large integration area. These shortcomings are motivations for researchers to find alternative solutions to obtain ALT effect.

Recently, great attention has been paid to the ALT effects in reciprocal, passive, and linear systems. Here, only

*mirbek.turduev@tedu.edu.tr by breaking the spatial symmetry of the linear and timeinvariant system, desired mode conversion can be achieved, which provides high transmission asymmetry between the forward and backward directions. ALT effect can be achieved by using reciprocal systems including conventional photonic crystals (PCs) [12-16], grating structures [17-19], mode order converters [20,21], metasurfaces [22,23], and chiral metamaterials $[24,25]$. Another interesting approach to achieve ALT effect is to use artificial materials that exhibit zero refractive index [26-29]. It is also possible to obtain zero refractive index by periodic PCs that exhibit simultaneous zero permittivity and permeability at the accidentally degenerated particular frequency called the Dirac-like point [30-33]. It should be noted that our proposed approach to achieve ALT effect does not violate the reciprocity theorem [3]. Reciprocity means a complete symmetry of the forward-backward couplings between the same propagation mode channels. The asymmetry in transmission achieved in this paper regards the propagation of the incident plane waves, which project into different modes of the photonic structure and connect different mode channels for forward and backward waves, therefore ALT effect does not violate the reciprocity.

The Dirac cone and semi-Dirac cone (SDC) dispersions are encountered by examining the intersections of photonic bands of PCs at degenerate frequencies [30-34]. These conical shapes of dispersion ensure that all-dielectric PCs exhibit a zero refractive index behavior at a particular frequency of "accidental" degeneracy [30-39]. Although both Dirac-like and SDC dispersion relations possess 

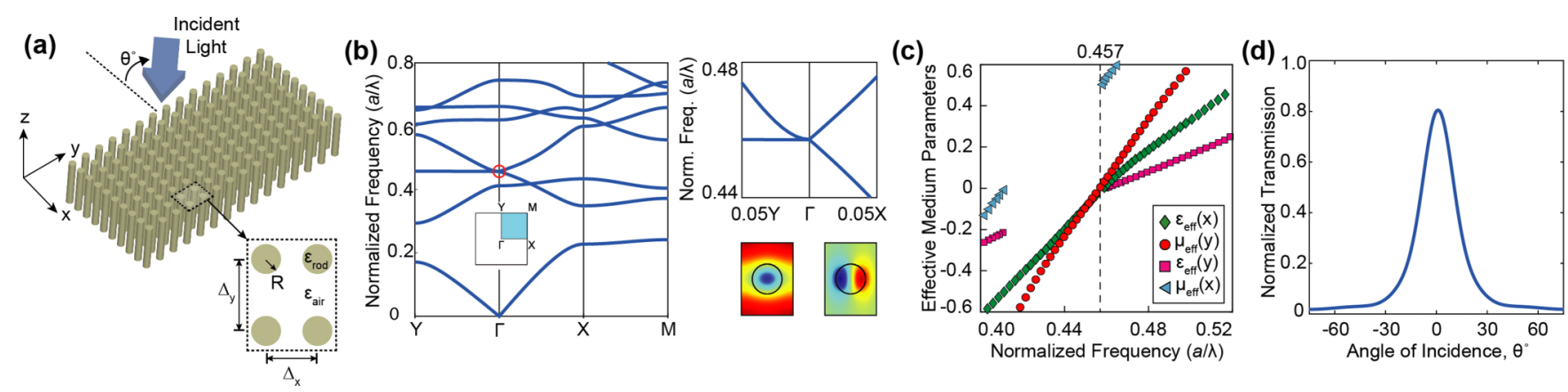

FIG. 1. (a) Three-dimensional representation of the rectangular lattice PC structure exhibiting SDC dispersion. (b) The band diagram of the corresponding PC structure for TM polarization. The zoomed inset shows a doubly degenerate state, i.e., SDC dispersion at the $\Gamma$ point, at the normalized frequency of $a / \lambda=0.457$, here the SDC dispersion that comprises a flat band, a quadratic band, and two linear bands. The electric-field eigenstates at the doubly degenerate point are also given as insets. (c) Effective medium parameters for both $\Gamma X$ and $\Gamma Y$ propagation directions of the proposed SDC PC. (d) Normalized transmission efficiency of the PC structure at SDC for different angles of incidence of the incident wave that propagated along the $\Gamma \mathrm{X}$ direction.

effective zero refractive index, the main difference lies behind the dual relation of relevant constituent parameters $\left(\varepsilon_{\mathrm{eff}}\right.$, $\left.\mu_{\text {eff }}\right)$. That is to say, PCs exhibiting Dirac-like cones show an impedance-matched epsilon-and-mu-near-zero (EMNZ $\rightarrow$ $\varepsilon_{\mathrm{eff}} \approx 0, \mu_{\mathrm{eff}} \approx 0$ ) characteristic along both transverse $(\Gamma \mathrm{Y})$ and longitudinal $(\Gamma \mathrm{X})$ propagation directions. On the other hand, the SDC dispersion relation ensures that PCs behave as an EMNZ material for one symmetry axis (e.g., $\Gamma X)$ and an impedance-mismatched epsilon-near-zero (ENZ $\rightarrow \varepsilon_{\text {eff }} \approx$ $\left.0, \mu_{\text {eff }} \neq 0\right)$ material for the transverse symmetry axis (e.g., $\Gamma Y)$. Here, the emerged accidental degeneracy phenomenon exhibits very interesting dispersion characteristics near the semi-Dirac point such as strong anisotropic wave transport due to EMNZ effect. Another interesting characteristic is that the light wave can propagate through the structure only in the case of normal incidence in the vicinity of degenerated frequency. This means that, by appropriately shaping the boundaries of the zero refractive index material, one can easily obtain ALT effect.

In this paper, we propose a unique technique to achieve ALT by incorporating evolutionary optimization with the SDC dispersion phenomenon. The evolutionary optimization algorithm known as differential evolution (DE) is applied to optimize the initially periodic PC structure exhibiting SDC dispersion effect. We report the application of an optimization algorithm to design a PC device based on SDC effect. It should also be noted that the idea of using an optimization approach to design a photonic structure attracts great attention $[40,41]$. To date, a variety of optimization algorithms has been proposed as a design approach to form unique optical devices based on PCs such as lenses [42], mode order converters [43], cavities [44], large photonic band-gap structures [45,46], optical couplers [47], and obtaining of a semi-Dirac-point [32].

The paper is organized as follows. First, in Sec. II, we perform plane-wave expansion (PWE) method for a rectangular lattice of cylindrical dielectric rods for designing a PC structure with a semi-Dirac point in photonic bands. Then, the integration of finite-difference time-domain (FDTD) method into the DE algorithm, which brought us to the ALT effect for two opposite directions of light, is presented. In Sec. III, the physical mechanisms for ALT are related with the scattered fields in the region of an intelligently distorted lattice and the excitation of the designed PC. Next, in Sec. IV, the numerical results of the designed PC structure are supported by experiments in the microwave regime, with a good agreement. Finally, Sec. V concludes the paper, by completing numerical and experimental results with physical interpretations.

\section{SEMI-DIRAC CONE DISPERSION AND ASYMMETRIC LIGHT TRANSMISSION}

The PC structure in this paper consists of a rectangular lattice of cylindrical dielectric rods in an air environment, as shown in Fig. 1(a). The relative permittivities of air background and dielectric alumina $\left(\mathrm{Al}_{2} \mathrm{O}_{3}\right)$ rods are selected as $\varepsilon_{\text {air }}=1.0$ and $\varepsilon_{\text {rod }}=9.80$, respectively. The dielectric rods have a radius of $R=0.29 a$, and the distances between the centers of rods are fixed to $\Delta x=1.0 a$ and $\Delta y=1.40 a$ for $x$ and $y$ directions, respectively, where $a$ scales the lattice constant. The rectangular lattice PC structure, the basis platform for optimization, consists of 10 and 15 columns in $x$ and $y$ directions, respectively. In Fig. 1(a), the corresponding PC is shown with structural parameters indicated in insets. In order to investigate the dispersion properties of the rectangular lattice PC structure, its band structure is numerically calculated for transverse-magnetic (TM) polarization, where magnetic field components are in the $x y$ plane $\left(H_{x}, H_{y}\right)$ and the electric-field component $\left(E_{z}\right)$ is perpendicular, by applying the PWE method [48]. As can be seen from the center of the Brillouin zone given in Fig. 1(b), the third and fourth TM polarization bands intersect at the normalized frequency of $a / \lambda=0.457$, where $\lambda$ denotes the wavelength in free space, and the intersection region is emphasized with a red-colored circle. This "accidental" doubly degenerate point is referred to as the semi-Dirac frequency where the periodic structure behaves as EMNZ and ENZ materials in the $\Gamma \mathrm{X}$ and $\Gamma \mathrm{Y}$ propagation directions $[31,32]$, respectively. Here, the term "accidental" originates from the fact that such dispersion exists only for certain structural parameters of a PC unit cell and does not appear as a result of symmetry. According to Ref. [31], there are certain requirements that the periodic photonic structure should satisfy in order to exhibit zero refractive index characteristics. First, at around the semi-Dirac point, the dispersion relation should consist of a 
(a)

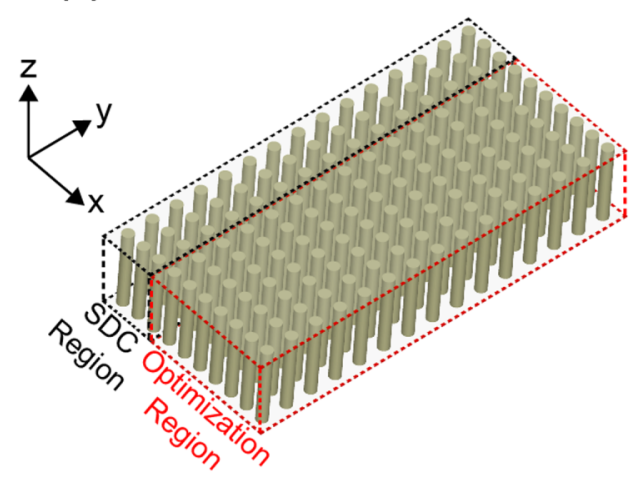

(b)

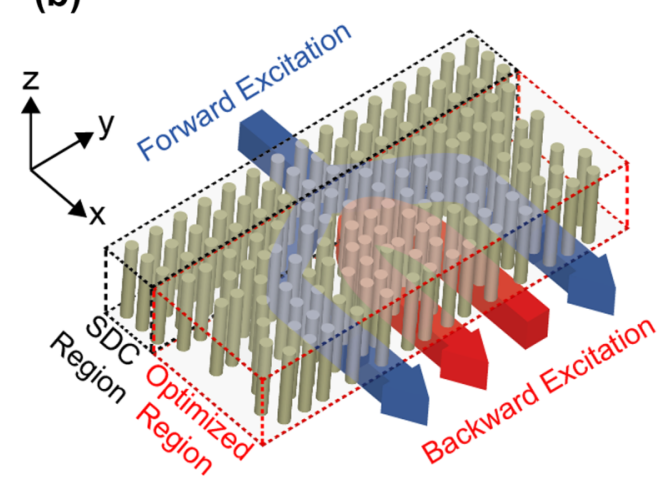

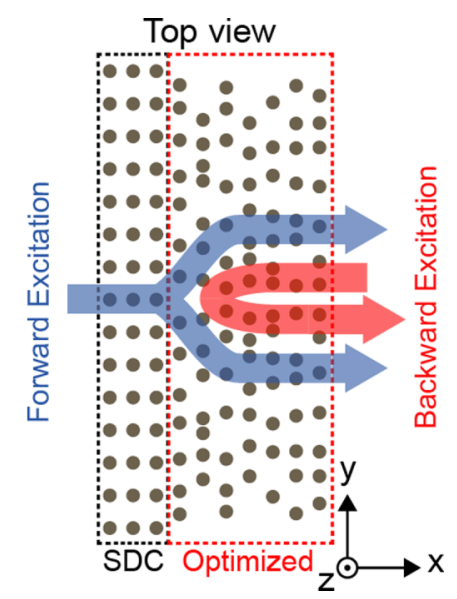

FIG. 2. (a) Three-dimensional representation of the PC structure, which is selected as a basis for optimization. The preselected fixed SDC region and the optimization region are surrounded by black and red dashed lines, respectively. (b) The optimized PC structure is given where the fixed SDC region and the optimized region are surrounded by black and red dashed lines, respectively. Forward and backward excitations of the optimized PC structure are shown by shaded blue and shaded red arrows, respectively.

flat band, a quadratic band, and two linear bands. Second, at the doubly degenerate frequency, electric-field eigenstates should comprise a monopolar state and a dipolar state. In order to explore SDC dispersion in detail, to see whether it meets the mentioned requirements, a magnified view of the doubly degenerated region is presented as an inset in Fig. 1(b). As can be seen, in the vicinity of the semi-Dirac frequency, dispersion curves consist of two linear bands for the $\Gamma \mathrm{X}$ propagation direction and also consist of a flat and a parabolic band along the $\Gamma Y$ propagation direction. Furthermore, eigenstates of the third and fourth dispersion bands at the doubly degenerate point are also given as insets in Fig. 1(b). As can be deduced from the electric-field profiles, the presented SDC dispersion relation arises by accidental degeneracy of monopolar and dipolar modes.

In order to investigate the constitutive relations of the proposed PC structure, PWE method is used to extract effective medium parameters [see Fig. 1(c)]. As one can see, effective optical parameters along the $\Gamma X$ symmetry axis $\left[\varepsilon_{\mathrm{eff}}(x), \mu_{\mathrm{eff}}(y)\right]$ intersect at the frequency of the semi-Dirac point $(a / \lambda=0.457)$ and are equal to the value of zero $\left[\varepsilon_{\mathrm{eff}}(x) \approx 0, \mu_{\mathrm{eff}}(y) \approx 0\right]$ at this frequency, which validates that the given medium shows an EMNZ characteristic along $\Gamma X$. On the other hand, for the $\Gamma Y$ propagation direction, only effective permittivity converges to zero $\left[\varepsilon_{\text {eff }}(y) \approx 0\right]$ at the semi-Dirac frequency and the effective permeability is not equal to zero $\left[\mu_{\mathrm{eff}}(x) \neq 0\right.$ ], which corresponds to the ENZ phenomenon for a given medium along the $\Gamma Y$ propagation direction.

As is known, the reflection of electromagnetic waves upon a zero index material (ZIM) critically depends on the incident angle of light. When Snell's law of refraction is applied to the air-ZIM interface $\left(n_{\text {air }} \theta_{\text {incident }}=n_{\text {ZIM }} \theta_{\text {refracted }}\right)$, the acceptance angle of ZIMs is calculated to be $\theta_{\text {incident }}=0^{\circ}$ since the refractive index of ZIM is equal to zero, $n_{\mathrm{ZIM}}=0$. In other words, $\theta_{\text {incident }}$ must be $0^{\circ}$ in order to satisfy the equation of Snell's law of refraction. Therefore, $\theta_{\text {incident }}=0^{\circ}$ can be defined as a critical angle for a perfect ZIM [34]. Thus, incidence at the nonzero angle results in a rapid decrease of transmission efficiency and in a strong reflection of the incident light. The periodic structure is sequentially excited by TM polarized Gaussian sources with different incident angles operating at the semi-Dirac frequency of $a / \lambda=0.457$. The transmission efficiency is measured at the exit surface of the PC structure. The dependence of the transmission on angle of incidence is shown in Fig. 1(d) where increase of the angle evidently decreases the forward transmission of the light. The corresponding angle sensitivity of the transmission can be associated with particular dispersion of an EMNZ characteristic along the $\Gamma X$ direction in the SDC PC structure. As can be seen from Fig. 1(b), the semi-Dirac point appears at the $\Gamma$ point.

The band gaps emerge only under oblique incidence and the light can penetrate through the structure only under normal incidence. This angle-sensitivity dispersion of the SDC PC structure motivated us to design an all-dielectric ALT device by using the optimization approach. The main goal of the paper is to generate efficient and compact grating like a medium behind the SDC PC region to achieve asymmetry between forward and backward light transmissions through the optimized PC medium at the semi-Dirac frequency of $a / \lambda=0.457$.

In this paper, we applied the DE algorithm [49] to achieve asymmetric light transmission in a PC structure that exhibits the SDC dispersion phenomenon. Specifically, the SDC PC structure subjected to optimization consists of ten columns of dielectric rods is shown in Fig. 2(a). In order to employ the excitement condition of the EMNZ characteristic of the SDC PC medium at normal incidence, three columns of the SDC PC structure (SDC region) are fixed throughout the optimization process, and seven remaining columns are considered as an "optimization region" to obtain ALT effect. The DE algorithm intelligently determines the positions of dielectric rods of the optimization region along the transverse $y$ axis. To prevent the overlapping of dielectric rods in a column, minimum and maximum distances between rods along the $y$ direction are fixed to $\Delta y_{\min }=0.60 a$ and $\Delta y_{\max }=2.0 a$, respectively. The optimization algorithm is integrated with two-dimensional 

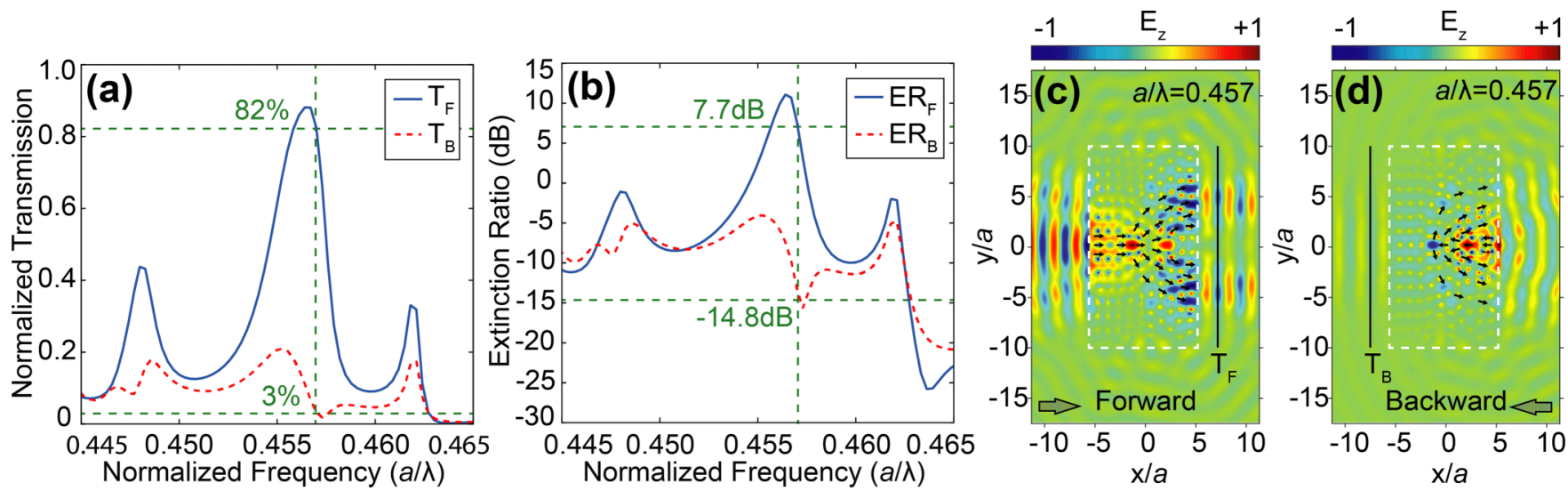

FIG. 3. (a) Normalized transmission efficiencies for forward $\left(T_{F}\right)$ and backward $\left(T_{B}\right)$ excitations of the optimized PC structure are represented as blue solid line and red dashed lines, respectively. (b) Extinction ratio values are calculated for forward (ER ${ }_{F}$ ) and backward $\left(\mathrm{ER}_{B}\right)$ excitations and plotted as blue solid line and red dashed lines, respectively. The vertical dashed green lines indicate the position of the semi-Dirac frequency of $a / \lambda=0.457$ on plots (a) and (b). The steady-state electric-field $\left(E_{z}\right)$ distributions of the optimized PC structure for forward and backward excitations and shown in (c) and (d), respectively, at the semi-Dirac frequency of $a / \lambda=0.457$. Dashed regions indicate the boundaries of the optimized PC structure and large arrows show the directions of excitations. Vertical lines superimposed to electric-field distribution plots denote the monitors for calculating forward and backward transmission efficiencies. Streamlines of electric fields are represented by small arrows.

FDTD method [51] and normalized transmission efficiencies are calculated for forward and backward directions at the semi-Dirac frequency of $a / \lambda=0.457$. Throughout the optimization process, the DE algorithm iteratively minimized the predefined cost function to obtain ALT effect as follows:

$$
f_{\cos t}=1-T_{F}+T_{B}
$$

where $T_{F}$ and $T_{B}$ denote the normalized transmission efficiencies of forward and backward directions, respectively, for the iteratively designed PC structure during the optimization process. The optimization was manually terminated when the algorithm converged to a good solution and did not further improve for several iterations.

It is important to note that the optimization algorithms do not search the entire solutions space but try to find a possible good candidate solution according to the defined cost function. As a result, the obtained solutions do not imply that it is the global minimum of the problem. On the other hand, the number of design parameters and a multiobjective cost function may affect the convergence performance of the algorithm, which is still superior to the brute-force sweep of the design parameters. The main purpose of applying an optimization algorithm is to acquire a solution which provides the desired properties for the system, not to search for the global minimum of the solution space.

The perspective and top views of the designed compact ALT PC structure are depicted in Fig. 2(b) where the blue and red arrows represent the forward transmission and backward reflection, respectively. The lateral and longitudinal sizes of the optimized PC structure are equal to $L_{x}=9.58 a$ and $L_{y}=$ $20.18 a$, respectively. The optimized PC structure is excited by a Gaussian source for both forward and backward directions. In order to calculate the transmission efficiencies, the monitors are placed at the exits of the optimized PC structure. In Fig. 3(a), the calculated normalized transmission efficiencies for forward and backward excitations are plotted. As can be seen, an ALT characteristic is obtained at the vicinity of the semi-Dirac frequency. The transmission efficiencies of forward and backward excitations are calculated as $T_{F}=$ $82 \%$ and $T_{B}=3 \%$, respectively, at the operating frequency of $a / \lambda=0.457$, which is indicated by the vertical dashed line in the same plot in Fig. 3(a). If we define an operating bandwidth of the designed PC structure by restricting the forward transmission efficiency to be higher than $75 \%$, it covers the normalized frequencies between $a / \lambda=0.4555$ and 0.4571 , which equal to a bandwidth of $3.5 \%$. Also, the backward transmission efficiency is below $20 \%$ in the determined bandwidth.

In addition, to evaluate the performance of the induced ALT effect, we calculated the extinction ratios for both forward $\left(\mathrm{ER}_{F}\right)$ and backward $\left(\mathrm{ER}_{B}\right)$ excitations and plotted the results in Fig. 3(b). The extinction ratio of forward excitation can be mathematically represented as follows:

$$
\mathrm{ER}_{F}=10 \times \log _{10}\left(T_{F} / R_{F}\right)
$$

where the $R_{F}$ denotes the forward reflection efficiency. Analogously for backward excitation,

$$
\mathrm{ER}_{B}=10 \times \log _{10}\left(T_{B} / R_{B}\right) .
$$

The calculated ER values of forward and backward excitations are equal to $\mathrm{ER}_{F}=7.7 \mathrm{~dB}$ and $\mathrm{ER}_{B}=-14.8 \mathrm{~dB}$, respectively, as indicated in Fig. 3(b). The calculated positive $\mathrm{ER}_{F}$ indicates high transmission efficiency along with low reflection for forward excitation whereas high reflection with very low transmission efficiency in backward excitation results in a negative $\mathrm{ER}_{B}$ value.

In order to get a picture of light distribution within the ALT PC structure at the semi-Dirac frequency, we depicted the numerically calculated steady-state electric-field $\left(E_{z}\right)$ distributions for forward and backward excitations in Figs. 3(c) and 3(d), respectively. The FDTD simulation region is surrounded by perfectly matched layers during the optimization process [50]. In Fig. 3(c), the light at normal incidence illuminates the optimized PC structure from its SDC region side and 
(a)
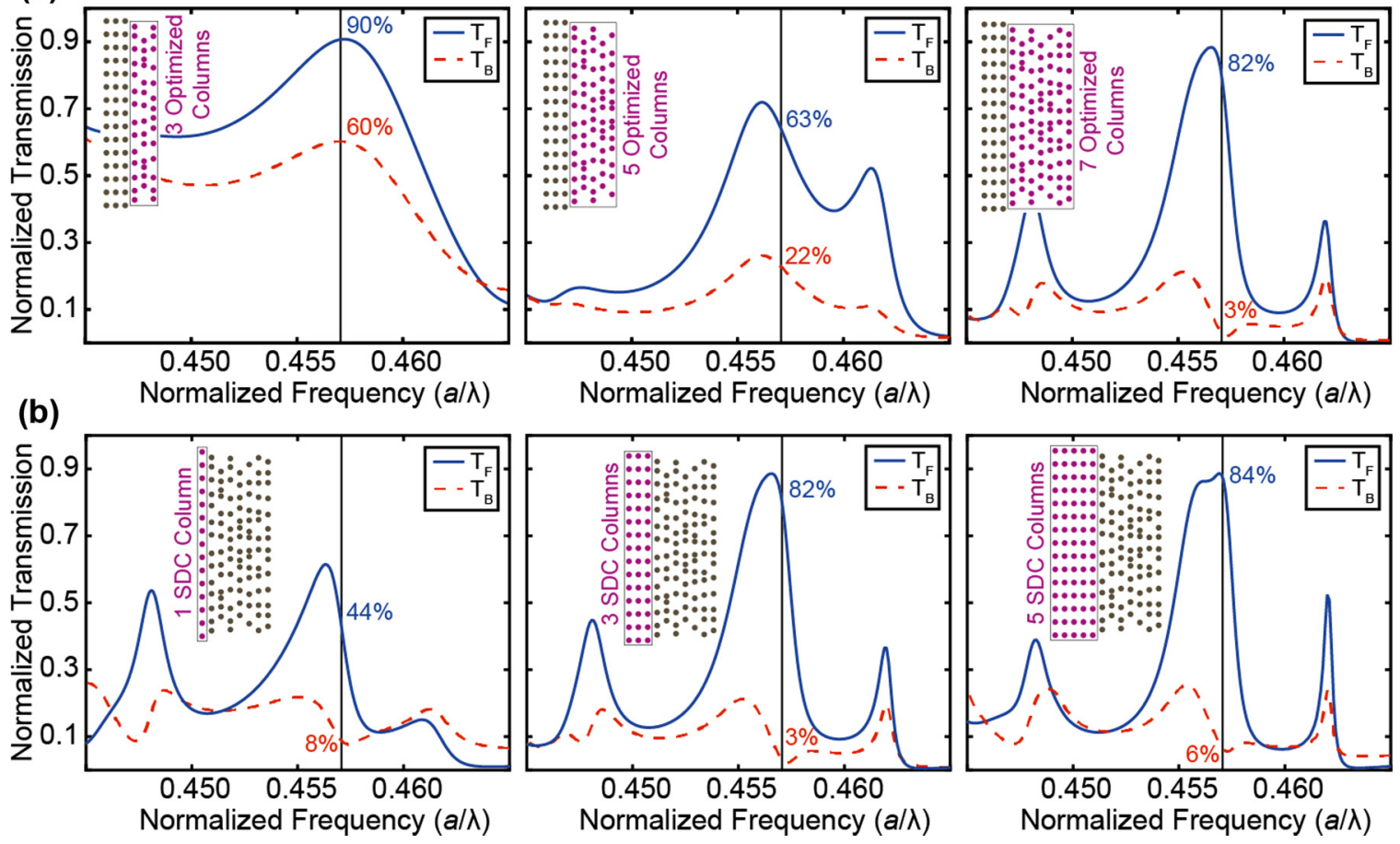

FIG. 4. (a) The impact of the number of columns of the optimized region (purple colored consecutive columns of the optimized region are added to the structure step by step) on forward and backward transmission efficiencies where the number of columns of the SDC region is fixed to three adjacent columns. (b) Forward and backward transmission efficiency variations according to the change of number of consecutive columns of the SDC region where the optimized region is kept unchanged. Top views of the PC structure for different cases are given as insets in figures. The semi-Dirac frequency of $a / \lambda=0.457$ is indicated in each transmission plot by a vertical solid black line and corresponding transmission values at that frequency are depicted in the same plots.

propagates with a low reflection at the surface through the SDC region along the $x$ axis. As the light enters the optimized region, due to incident angle sensitivity of the SDC PC medium at the semi-Dirac point, the light is scattered and divided into two branches while exiting the structure. Still, the transmission efficiency is as high as $82 \%$ as shown in Fig. 3(a). In contrast, as can be seen in Fig. 3(d) for the backward excitation case, the incident light enters from the DE optimized region and seems to be guided around the $x$ axis. When the incident light with planar wave fronts enters the optimized structure, it starts to scatter due to irregularly distributed dielectric rods. Hence, wave fronts of propagating light inside the optimized region become fairly distorted. As a result, propagating light through the $\mathrm{DE}$ region cannot enter the SDC region due to nonzero angle of incidence and reflects back from the SDC PC surface. Thus, we can observe ALT effect at the output of the optimized PC structure with forward and backward transmissions of 82 and 3\%, respectively.

Although the asymmetric light propagation mechanism proposed in Ref. [27] exploits a similar concept, which is based on highly reflecting diffraction orders from a ZIM surface, there is still an additional important difference between the two papers. Here, the main difference is attributed to the type of conical dispersion relations that PC structures possess. The proposed PC structure used in Ref. [27] exploits the Dirac-like cone phenomenon, in which the periodic medium behaves as an impedance-matched SDC medium along both transverse propagation directions ( $x$ and $y$ directions). In general, Dirac-like cone-based PC devices require reflective boundaries, e.g., perfect electric conductors (PECs), to return the light propagation along undesired directions [30]: if one does not use PEC-like materials to prevent power leakage, transmission efficiency of the photonic devices may dramatically reduce. On the other hand, differently from the concept given in Ref. [27], we utilize the anisotropic dispersive nature of the SDC phenomenon, which ensures that a periodic medium behaves as an impedance-matched EMNZ material and an impedance-mismatched ENZ material for different propagation directions. For the PC structure given in Fig. 1(a), corresponding effective parameters of the periodic medium are not equal to zero simultaneously $\left(\varepsilon_{\text {eff }} \approx\right.$ $0, \mu_{\text {eff }} \neq 0$ ) along the $y$ direction. This characteristic ensures that the optical impedance along the $y$ direction has larger values than air since $Z=\mu / \varepsilon$. Furthermore, thanks to the impedance mismatch between air and ZIM interfaces, the light transmission efficiency decreases along the $y$ direction. Thus, the proposed PC structure does not require reflective boundaries along the $y$ direction, since the electromagnetic waves propagating inside the $\mathrm{PC}$ structure are confined along the $y$ direction due to ENZ medium effect and can only advance through the $x$ direction due to EMNZ medium effect. Here, the self-confinement of light inside an asymmetric light transmission device may ease the implementation process of photonic applications by reducing the requirements of zero refractive index PCs to be utilized. 


\section{DISCUSSION ON THE TRANSMISSION RESPONSE OF THE OPTIMIZED ALT DEVICE BASED ON THE SEMI-DIRAC CONE DISPERSION PHENOMENON}

Here the ALT mechanism of the optimized PC structure is investigated in further detail. As stated previously, the designed structure has two regions: the SDC region and the optimized region. During the optimization process, the DE algorithm optimized all seven columns of the optimization region while keeping the SDC region fixed. In order to carefully inspect and understand the impacts of each column on ALT effect, we sequentially added the columns of the optimized region one by one while keeping the three columns of the SDC region fixed and measured the transmission response of the $\mathrm{PC}$ structure. In a similar manner, the effect in the SDC region of the different number of columns on transmission efficiencies is also investigated for both forward and backward excitations. The corresponding transmission efficiencies are plotted in Fig. 4. By fixing the SDC region, we added the columns of the optimized region starting from left to right along the $x$ axis one by one and plotted the corresponding transmission measurements for forward and backward excitations in Fig. 4(a). The sequentially added columns are indicated in rectangular frames and their schematic representations are given as insets in the corresponding plots. As can be seen from Fig. 4(a), the transmission efficiencies at the semi-Dirac frequency change as the columns of the optimized region are added to the structure. In the plot on the left in Fig. 4(a), one can see the transmission efficiency plots of forward and backward excitations for the case when three columns of the optimization region were added. Here, the values of both forward and backward transmissions are relatively high, which means that there is a small contrast between them. Placing an additional two columns increases the contrast between forward and backward transmissions, which can be seen from the plot in the middle in Fig. 4(a). In other words, five optimization columns decrease the backward transmission from 60 to $22 \%$ but also decrease the forward transmission from 90 to $60 \%$. Finally, all seven columns give us the desired result that the forward transmission increases to $82 \%$ whereas backward transmission drops down to $3 \%$ as shown in the plot on the right in Fig. 4(a). In this regard, we can say that the implemented optimization procedure achieved the desired ALT performance by optimizing only seven columns of the SDC PC structure.

The effect of the SDC region on transmission efficiencies for both forward and backward propagation is shown in Fig. 4(b). As can be seen the transmission efficiencies are low for forward and backward excitations in the case of a single SDC PC column. On the other hand, if we place a second and third column in the SDC region of the PC structure, an essential increase in forward transmission and abrupt drop in backward transmission can be observed as can be seen from the plots in the middle of Fig. 4(b). Compared to a single SDC column, the periodically placed three columns start to behave as a SDC PC medium and show its effect on the ALT PC structure. In the plot on the right of Fig. 4(b), we added fourth and fifth columns in the SDC region to the configuration and we observed that transmission efficiency is slightly increased for forward excitation from 82 to $84 \%$, however there occurs a considerable increment of transmission efficiency for backward excitation from 3 to $6 \%$. Here, the SDC region surprisingly increases the transmission efficiency of the optimized region for forward excitation, i.e., the SDC region performs as an antireflection coatinglike behavior for the optimized region in forward excitation. Since the main task is to design a compact and efficient ALT PC device, the three columns of the SDC region are considered to be sufficient.

For different numbers of columns in the optimized region, the steady-state electric-field $\left(E_{z}\right)$ distributions are calculated and given in Fig. 5. In order to better understand the corresponding field distributions, the transmission efficiency plots given in Fig. 4 should be considered along with the representations presented in Fig. 5. As can be seen from Fig. 5(a), three columns in the optimized region provide easy field penetration of the forward propagating light through the ALT structure whereas the transmission efficiency is still considerably high for the backward excitation. When we consequently placed an additional two optimized columns in the optimized region, transmission efficiency decreases for both forward and backward excitations as indicated in the middle plot of Fig. 4(a). Reduction of the transmission in both directions can also be deduced from the field distributions in Fig. 5(b). Here, one can see the localization of light at two different positions along the $x$ axis for forward direction excitation; the left one is closer to the SDC region and the right one is near to the edge of the PC structure. Moreover, one can see a reflection inside the optimized region around the localized light near the edge of the PC structure which may cause a decrease in transmission efficiency for the forward light propagation case. On the other hand, as is seen in the left plot in Fig. 5(b), the incident light weakly penetrates through the optimized region, i.e., high reflection occurs at the entrance of the PC structure for the backward excitation case.

Lastly, as we add two more optimized columns into the PC structure, the propagating light inside the PC structure is divided into the two separate branches around the light localization regions and is channeled to the back plane of the ALT structure as can be seen in the right plot in Fig. 5(c). For backward excitation, highly reflected light is similarly divided into two branches and propagation of light is blocked for propagation which causes the almost negligible transmission efficiency of backward light propagation as indicated in the left plot of Fig. 5(c). From the given field distributions for both forward and backward excitations, one can see that incident light is localized in two regions and channeled to the surface between air and the optimized region. These regions, where light localizes, can be considered as cavity defects. Hence, we think that the increment in forward transmission efficiencies of five and seven columns of the optimized region is the result of cavity enhancement, i.e., a cavity enhanced transmission takes place in forward excitation. On the other hand, for backward excitation, the incident light does not reflect from the surface between air and the optimized region. In contrast, incident light enters the structure and optimization induced cavity-based light localization diffracts the light and causes a scattering effect. Therefore, scattered light excites all $k$ vectors in the propagation direction, resulting in the blocking of light in backward penetration of the SDC PC 

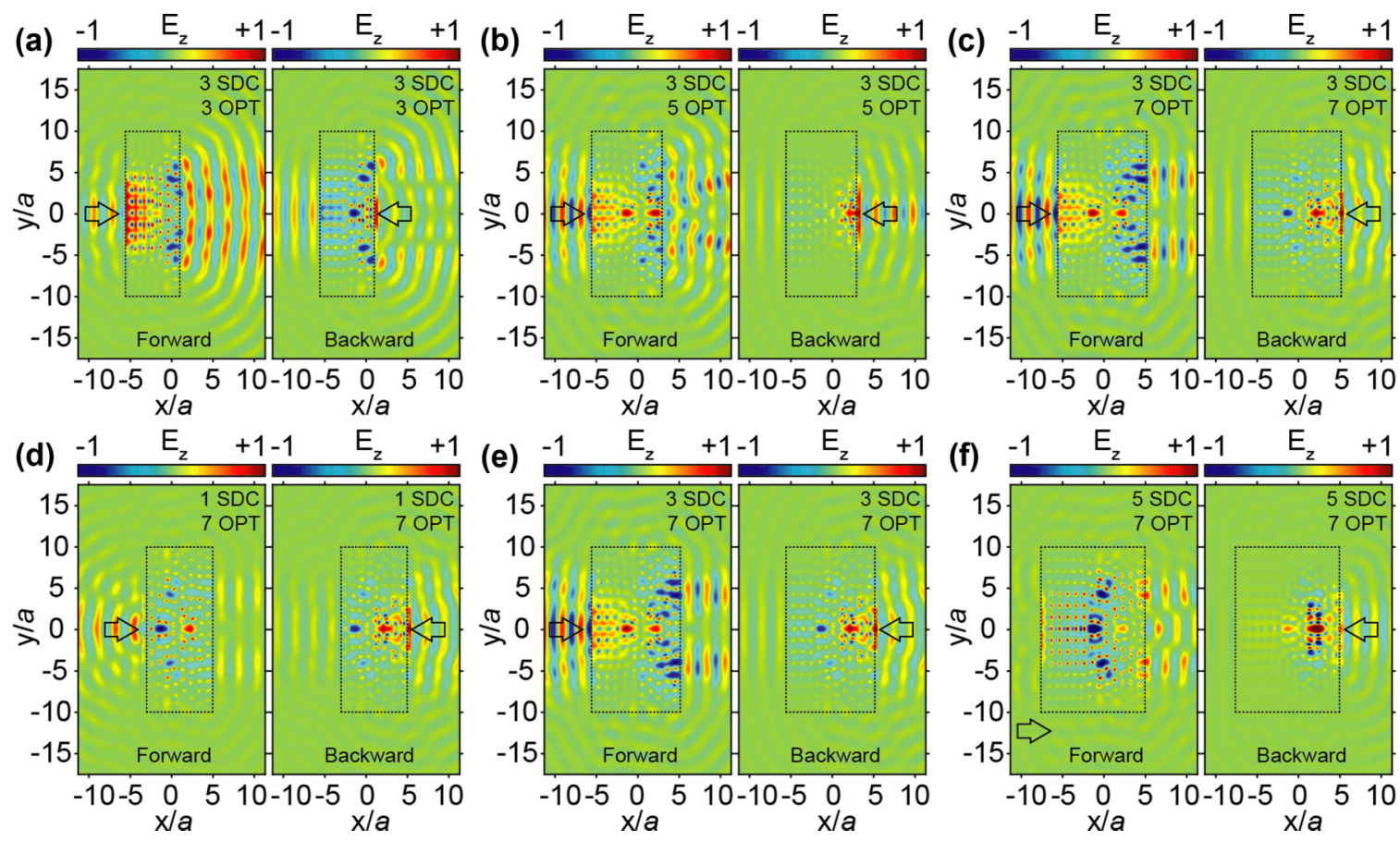

FIG. 5. For forward and backward excitations, steady-state electric-field $\left(E_{z}\right)$ distributions of PC structures consist of three SDC columns and (a) three, (b) five, and (c) seven columns of the optimized region are shown. Steady-state electric-field $\left(E_{z}\right)$ distributions of PC structures for forward and backward excitations where columns of the optimized region are fixed and the SDC region is changed to consist of (d) a single column, (e) three columns, and (f) five columns. Arrows indicate the direction of incident light and dashed regions surround the boundaries of corresponding PC structures under analysis. All steady-state electric-field distributions are calculated at an operating frequency of $a / \lambda=0.457$.

structure due to its inherent angle-sensitivity dispersion [see Fig. 1(d)] phenomenon at the semi-Dirac frequency point. As a result, backward illuminated light reflects back and this can be considered as the main mechanism for obtaining very low transmission efficiency for backward excitation.

It is also important to analyze the impact of the SDC region on ALT effect. For this reason, we investigated the field distributions of the PC structure changing the number of columns of the SDC region in Figs. 5(d)-5(f). In Fig. 5(d), only one column of the SDC region is considered where the optimization region is kept unchanged. Here, for forward excitation, the incident light mostly reflects from the input surface of the PC structure, which results in very low transmission of light. However, the incident light is localized and channeled to the input surface for the backward excitation case. The low transmission for both excitation directions appears because the SDC region does not provide a desired angle-sensitive transmission effect with a single column. Next, we sequentially added three columns in the SDC region where field distribution becomes intensified for the forward case and there still exists a low penetration of light in the backward direction as can be observed in the right and left plots of Fig. 5(e). It can be deduced from Fig. 5(e) that the transmission efficiency is increased for forward excitation and is decreased for backward excitation. Further, increasing the number of columns in the SDC region does not improve the ALT effect but increases the size of the PC structure as can be seen in Fig. 5(f).

Next, we numerically investigated the robustness of the designed photonic structure by using FDTD method. For this purpose, we introduce some random noise to the positions of dielectric rods in the optimized part of the designed photonic structure. Here, the positions of the corresponding rods are randomly changed along both $x$ and $y$ axes. First, we set the probability of shift for a rod as $30 \%$, which may be considered as an error rate of fabrication. Then, we separately define random shifts along $x$ and $y$ axes to be between $-0.05 a$ and $+0.05 a$ where the radius of dielectric rods is equal to $0.29 a$ as stated before. It should be noted that the shift values vary from one rod to another. Later, we formed several photonic structures and performed FDTD simulations to calculate both forward and backward transmission efficiencies of them. According to the numerical calculations, the forward transmission efficiencies stayed above $78 \%$ whereas the backward transmission efficiencies did not exceed the value of $10 \%$. Since the numerical results of transmission efficiencies were presented as 82 and 3\% for forward and backward excitation directions, respectively, the randomly distributed rod positions did not drastically worsen the asymmetric light transmission performance of the proposed photonic structure.

\section{EXPERIMENTAL VERIFICATION OF THE NUMERICALLY ANALYZED ALT EFFECT IN THE MICROWAVE REGIME}

The numerically demonstrated ALT effect is experimentally verified by performing the experiments in the microwave regime. The designed $\mathrm{PC}$ structure is prepared by periodically aligning alumina $\left(\mathrm{Al}_{2} \mathrm{O}_{3}\right)$ rods with a permittivity of $\varepsilon_{\text {rod }}=9.80$. The schematic view of the experimental setup is represented in Fig. 6(a). In order to generate and detect 

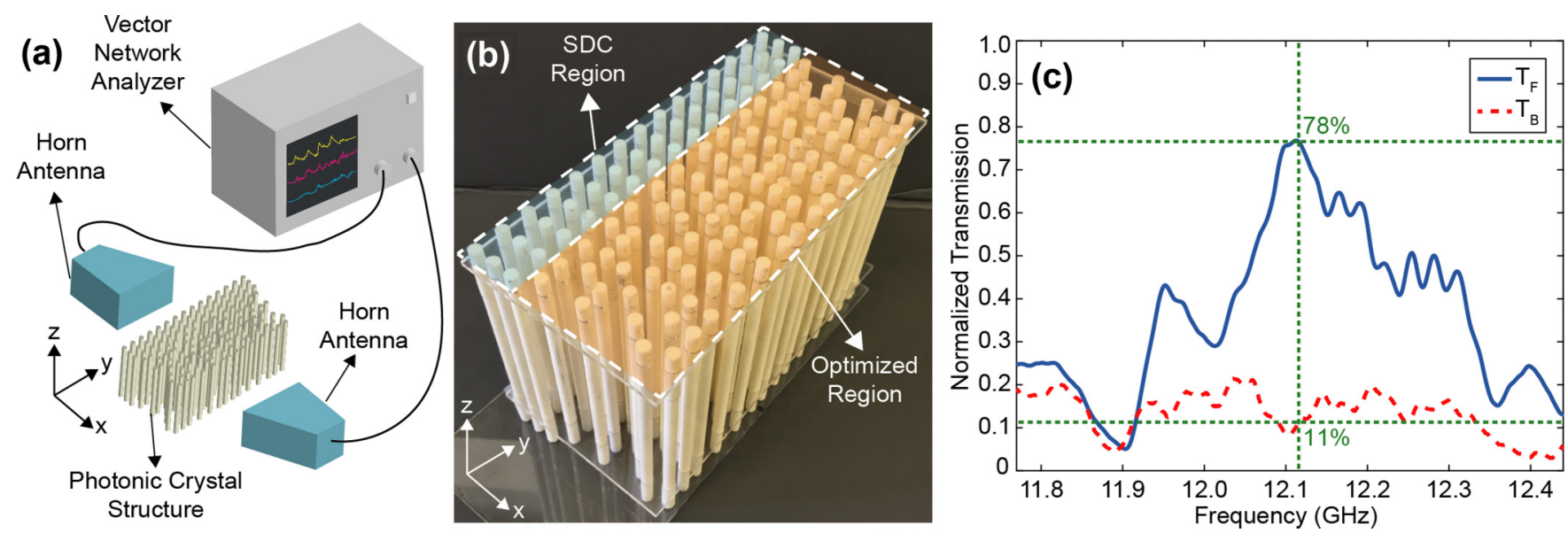

FIG. 6. (a) The schematic view of the microwave experimental setup to measure transmission efficiencies of the designed PC structure for both forward and backward excitations. (b) Illustration of the designed PC structure. (c) Measured transmission efficiencies of the PC structure for both forward (blue solid line) and backward (red dashed line) excitations are plotted.

microwaves, the Agilent E5071C ENA vector network analyzer is employed during the experiments. In microwave experiments, the lattice constant is fixed to $a=11.32 \mathrm{~mm}$ and corresponding diameters and heights of the alumina rods are equal to 6.60 and $152 \mathrm{~mm}$, respectively. The PC structure examined in microwave experiments consists of ten columns of alumina rods. Furthermore, the overall lateral and longitudinal sizes of the designed PC structure are 108.46 and $228.46 \mathrm{~mm}$, respectively. For given structural parameters, the semi-Dirac frequency is calculated as $12.11 \mathrm{GHz}$. The fabricated ALT PC structure is excited by using a horn antenna placed in front of it. The horn antenna produces Gaussian profiled microwaves and, in order to be in parallel with the excitation conditions of numerical calculations, the horn antenna is located at a distance of $20 \mathrm{~mm}$ away from the structure. Another identical horn antenna is placed at the back plane of the PC structure to measure the transmission efficiency of the structure. The photographic representation of the fabricated structure is given in Fig. 6(b). The measured forward and backward transmission efficiencies are superimposed in Fig. 6(c). Here, the forward transmission efficiency $\left(T_{F}\right)$ measured as $78 \%$ whereas backward transmission efficiency $\left(T_{B}\right)$ equals to $11 \%$ at the semi-Dirac frequency of $12.11 \mathrm{GHz}$.

In addition, to scan the electric-field $\left(E_{z}\right)$ distribution in the back plane of the ALT PC structure, a monopole antenna is employed where the schematic of the experimental setup for field measurements is represented in Fig. 7(a). Here, the monopole antenna is mounted on a motorized motioncontrolled scanning device that moves along both $x$ and $y$ directions to sweep the interested area. The monopole antenna scans the area having sizes of 50 and $220 \mathrm{~mm}$ in the $x$ direction and $y$ direction, respectively, starting $1 \mathrm{~mm}$ away from the ALT PC structure with 4-mm steps in both directions. For forward direction, numerically and experimentally obtained electric-field $\left(E_{z}\right)$ distributions and magnitudes of electricfield $\left(\left|E_{z}\right|\right)$ distributions are shown in Figs. 7(b) and 7(c), respectively. Similarly, for backward direction, calculated and measured electric-field distributions and their magnitudes are depicted in Figs. 7(d) and 7(e), respectively. Figure 7(b) and 7(c) exhibit good agreement between experimental and numerical data for forward excitation. The appearance of double lobes in the field distribution is apparent for both cases. On the other hand, overall field profiles for the backward direction in Figs. 7(d) and 7(e) do not match well. Such difference might be due to the inexact placement of the alumina rods in the irregular optimized region. Moreover, we should note that the amount of transmitted light is reduced in the backward direction compared with the forward one for both experimental and numerical results. Lastly, there are two different schematics depending on the excitation direction of the incident light where the ALT structure is flipped to realize different excitation directions.

Even though the mechanisms of high forward and low backward transmission efficiencies are explained as the scattering of incident light from the SDC region due to its dependency on angle of incidence, detailed clarification for the asymmetric light transmission of the proposed PC structure is still needed. Here, it is important to touch on the misuses of "optical isolation" and "unidirectional light transmission," which are different from asymmetric light transmission. According to the Ref. [3], the optical isolation can be defined as prohibiting transmission in backward direction for all possible modes that can be excited. For this reason, an "asymmetric scattering matrix," which should be valid for all modes, is required to obtain proper optical isolation. It should be noted that an asymmetric scattering matrix can be achieved by breaking the Lorentz symmetry (reciprocity) condition. In order to violate the reciprocity, i.e., to design nonreciprocal devices, metamaterials [2], magneto-optic materials [52], and indirect interband photonic structures [1] are proposed. Since linear and time-independent materials do not provide an asymmetric scattering matrix, it is not possible to design an optical isolator or an optical diode by using these types of materials. Furthermore, optical isolation, optical diode effect, and unidirectional transmission of light are obtained via alldielectric designs where the functionalities of the structures are limited to a finite number of excited modes or certain diffraction mode [12,13,27,53,54]. However, it is considered to be controversial to use the term "optical isolation" or "unidirectional light transmission" for designs made of only 
(a)

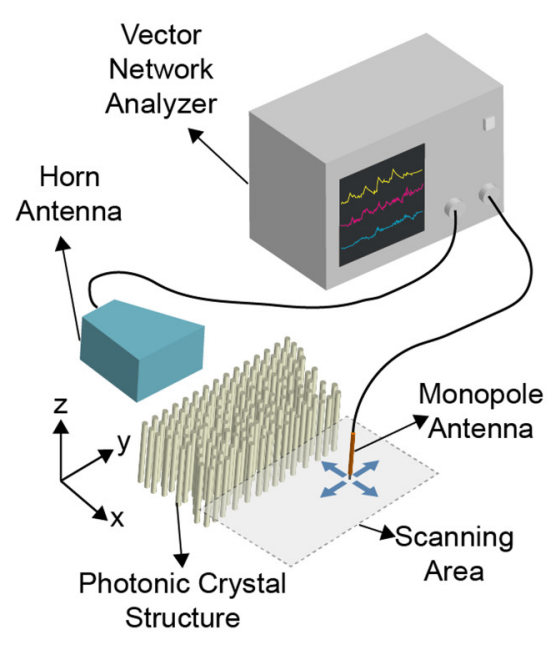

(b)

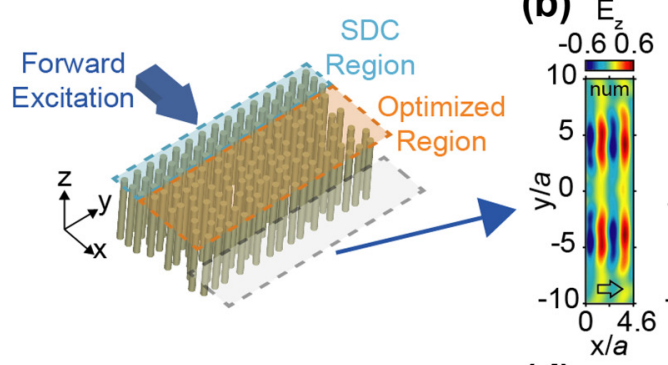

(d) $E_{2}$

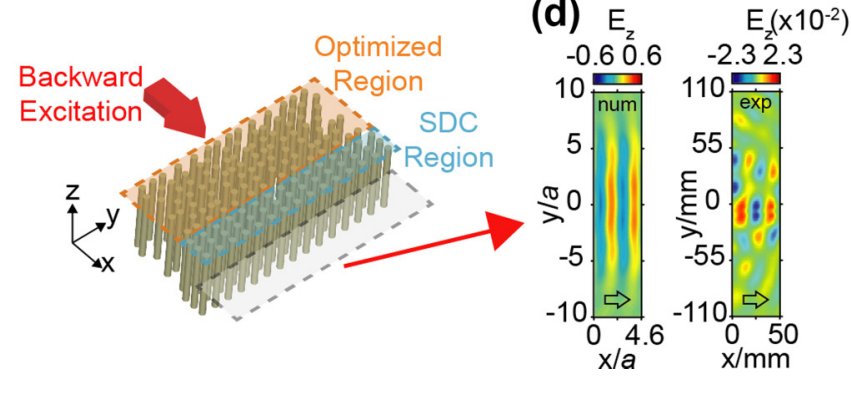

(c) $E_{\mathrm{E}}$
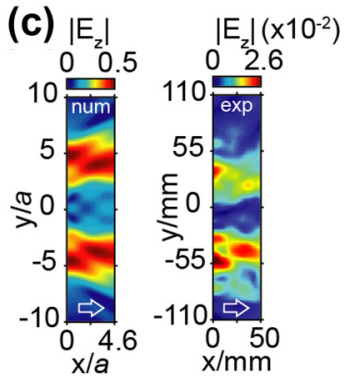

(e) $\mathrm{E}$
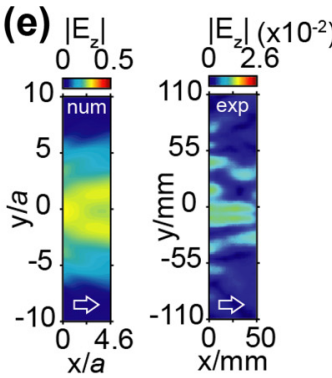

FIG. 7. (a) The schematic view of the microwave experimental setup to scan field distribution behind the designed PC structure for both forward and backward excitations. Numerically calculated and experimentally measured (b) electric-field $\left(E_{z}\right)$ distributions and (c) magnitude of electric-field $\left(\left|E_{z}\right|\right)$ distributions are represented for forward excitations. Numerically calculated and experimentally measured (d) electricfield $\left(E_{z}\right)$ distributions and (e) magnitude of electric-field $\left(\left|E_{z}\right|\right)$ distributions are represented for backward excitations.

dielectric material as discussed in Ref. [3]. On the other hand, using the term "asymmetric light transmission" is not in conflict with the reciprocity condition since asymmetric light transmission does not require an asymmetric scattering matrix [3]. Hence, we claim that our optimized PC structure provides an ALT effect and we are not claiming that the designed structure is an optical isolator because its operation is restricted to the fundamental mode of excitation for both forward and backward directions.

\section{CONCLUSION}

In conclusion, the asymmetric light transmission is obtained in a PC with the SDC dispersion phenomenon via the DE algorithm, which is due to particular positioning of alumina rods in a predefined optimization region along the $y$ direction. According to the numerical analysis of the designed PC structure by FDTD method, forward and backward transmission efficiencies are calculated as 82 and 3\%, respectively, at the operating normalized frequency of $a / \lambda=0.457$, which is the frequency of SDC dispersion. Even though ALT effect is optimized for a single frequency, the structure provides an unequal amount of transmitted light for other wavelengths at the neighborhood of the targeted wavelength. The physical mechanism of the designed asymmetric light transmission is the following: the distorted wave fronts from the irregular part of the photonic structure broaden the angular distribution of the backward incident wave, and the large angle components efficiently reflect from the interface between the regular and optimized part of the photonic structure. Large angle components do not couple into the zero index material efficiently. On the other hand, the waves outcouple from the zero index material to the optimized region quite efficiently. This is the main physical principle of the asymmetry. The optimization procedure, explored in detail in this paper, allows enhancing this asymmetry to its maximum values by, respectively, increasing and reducing the scattering in the optimized area for backward and forward waves. Furthermore, we performed experiments in the microwave regime to verify the presented numerical results. A good agreement between experimental and numerical results is obtained. At the frequency of $12.11 \mathrm{GHz}$, transmission efficiencies are measured as 78 and $11 \%$ for forward and backward excitations, respectively. It is important to note that the optimization algorithm is applied to design a photonic device based on SDC dispersion.

\section{ACKNOWLEDGMENTS}

This work was supported by the Scientific and Technological Research Council of Turkey (TUBITAK) under Project No. 116F182. H.K. acknowledges partial support from the Turkish Academy of Sciences.
[1] Z. Yu and S. Fan, Complete optical isolation created by indirect interband photonic transitions, Nat. Photon. 3, 91 (2009).

[2] L. Bi, J. Hu, P. Jiang, D. H. Kim, G. F. Dionne, L. C. Kimerling, and C. A. Ross, On-chip optical isolation in monolithically integrated non-reciprocal optical resonators, Nat. Photon. 5, 758 (2011).
[3] D. Jalas, A. Petrov, M. Eich, W. Freude, S. Fan, Z. Yu, R. Baets, M. Popovic, A. Melloni, J. D. Joannopoulos, M. Vanwolleghem, C. R. Doerr, and H. Renner, What is- and what is not- an optical isolator, Nat. Photon. 7, 579 (2013).

[4] J. Y. Chin, T. Steinle, T. Wehlus, D. Dregely, T. Weiss, V. I. Belotelov, B. Stritzker, and H. Giessen, Nonreciprocal 
plasmonics enables giant enhancement of thin-film faraday rotation, Nat. Commun. 4, 1599 (2013).

[5] M. Soljacic and J. D. Joannopoulos, Enhancement of nonlinear effects using photonic crystals, Nat. Mater. 3, 211 (2004).

[6] K. Gallo, G. Assanto, K. R. Parameswaran, and M. M. Fejer, All optical diode in a periodically poled lithium niobate waveguide, Appl. Phys. Lett. 79, 314 (2001).

[7] R. Philip, M. Anija, C. S. Yelleswarapu, and D. V. G. L. N. Rao, Passive all-optical diode using asymmetric nonlinear absorption, Appl. Phys. Lett. 91, 141118 (2007).

[8] L. Feng, M. Ayache, J. Huang, Y. Xu, M. Lu, Y. Chen, Y. Fainman, and A. Scherer, Nonreciprocal light propagation in a silicon photonic circuit, Science 333, 729 (2011).

[9] L. Fan, J. Wang, L. T. Varghese, H. Shen, B. Niu, Y. Xuan, A. M. Weiner, and M. Qi, An all-silicon passive optical diode, Science 335, 447 (2012).

[10] H. Dotsch, N. Bahlmann, O. Zhuromskyy, M. Hammer, L. Wilkens, R. Gerhardt, P. Hertel, and A. F. Popkov, Applications of magneto-optical waveguides in integrated optics: Review, J. Opt. Soc. Am. B 22, 240 (2005).

[11] M. Vanwolleghem, X. Checoury, W. Smigaj, B. Gralak, L. Magdenko, K. Postava, B. Dagens, P. Beauvillain, and J. Lourtioz, Unidirectional band gaps in uniformly magnetized two-dimensional magnetophotonic crystals, Phys. Rev. B 80, 121102(R) (2009).

[12] C. Wang, X. L. Zhong, and Z. Y. Li, Linear and passive silicon optical isolator, Sci. Rep. 2, 674, (2012).

[13] A. Cicek, M. B. Yucel, O. A. Kaya, and B. Ulug, Refractionbased photonic crystal diode, Opt. Lett. 37, 2937 (2012).

[14] C. Lu, X. Hua, Y. Zhang, Z. Li, X. Xu, H. Yang, and Q. Gonga, Ultralow power all-optical diode in photonic crystal heterostructures with broken spatial inversion symmetry, Appl. Phys. Lett. 99, 051107 (2011).

[15] A. E. Serebryannikov, One-way diffraction effects in photonic crystal gratings made of isotropic materials, Phys. Rev. B 80, 155117 (2009).

[16] Y. Zhang, Q. Kan, and G. P. Wang, One-way optical transmission in silicon grating-photonic crystal structures, Opt. Lett. 39, 4934 (2014).

[17] S. Cakmakyapan, A. E. Serebryannikov, H. Caglayan, and E. Ozbay, One-way transmission through the subwavelength slit in nonsymmetric metallic gratings, Opt. Lett. 35, 2597 (2010).

[18] M. Stolarek, D. Yavorskiy, R. Kotynski, C. J. Z. Rodríguez, J. Łusakowski, and T. Szoplik, Asymmetric transmission of terahertz radiation through a double grating, Opt. Lett. 38, 839 (2013).

[19] H Gao, Z. Y. Zheng, J. J. Dong, J. Feng, and J. Zhou, Multifrequency optical unidirectional transmission based on oneway guided mode resonance in an extremely simple dielectric grating, Opt. Commun. 355, 137 (2015).

[20] V. Liu, D. A. B. Miller, and S. Fan, Ultra-compact photonic crystal waveguide spatial mode converter and its connection to the optical diode effect, Opt. Express 20, 28388 (2012).

[21] F. Callewaert, S. Butun, Z. Li, and K. Aydın, Inverse design of an ultra-compact broadband optical diode based on asymmetric spatial mode conversion, Sci. Rep. 6, 32577 (2016).

[22] B. Shen, R. Polson, and R. Menon, Broadband asymmetric light transmission via all-dielectric digital metasurfaces, Opt. Express 23, 20961 (2015).
[23] L. Zhang, S. Mei, K. Huang, and C. W. Qiu, Advances in full control of electromagnetic waves with metasurfaces, Adv. Opt. Mater. 4, 818 (2016).

[24] C. Menzel, C. Helgert, C. Rockstuhl, E. Kley, A. Tunnermann, T. Pertsch, and F. Lederer, Asymmetric Transmission of Linearly Polarized Light at Optical Metamaterials, Phys. Rev. Lett. 104, 253902 (2010).

[25] G. Kenanakis, A. Xomalis, A. Selimis, M. Vamvakaki, M. Farsari, M. Kafesaki, C. M. Soukoulis, and E. N. Economou, Three-dimensional infrared metamaterial with asymmetric transmission, ACS Photonics 2, 287 (2015).

[26] Y. S. Zhou, L. M. Zhao, S. Lan, and H. Wang, An optical one-way device constructed with an epsilon-near-zero prism inserted in a metal slit, EPL 109, 54003 (2015).

[27] H. Gao, Y. Zhou, and Z. Zheng, Broadband unidirectional transmission realized by properties of the dirac cone formed in photonic crystals, J. Opt. 18, 105102 (2016).

[28] Y. Li, B. Liang, Z. Gu, X. Y. Zou, and J. C. Cheng, Unidirectional acoustic transmission through a prism with near-zero refractive index, Appl. Phys. Lett. 103, 053505 (2013).

[29] Y. Y. Fu, L. Xu, Z. H. Hang, and H. Y. Chen, Unidirectional transmission using array of zero-refractive-index metamaterials, Appl. Phys. Lett. 104, 193509 (2014).

[30] X. Huang, Y. Lai, Z. H. Hang, H. Zheng, and C. T. Chan, Dirac cones induced by accidental degeneracy in photonic crystals and zero-refractive-index materials, Nat. Mater. 10, 582 (2011).

[31] Y. Wu, A semi-Dirac point and an electromagnetic topological transition in a dielectric photonic crystal, Opt. Express 22, 1906 (2014).

[32] U. G. Yasa, M. Turduev, I. H. Giden, and H. Kurt, Full utilization of semi-dirac cones in photonics, Phys. Rev. B 97, 195131 (2018).

[33] P. Moitra, Y. Yang, Z. Anderson, I. I. Kravchenko, D. P. Briggs, and J. Valentine, Realization of an all-dielectric zero-index optical metamaterial, Nat. Photon. 7, 791 (2013).

[34] J. W. Dong, M. L. Chang, X. Q. Huang, Z. H. Hang, Z. C. Zhong, W. J. Chen, Z. Y. Huang, and C. T. Chan, Conical Dispersion and Effective Zero Refractive Index in Photonic Quasicrystals, Phys. Rev. Lett. 114, 163901 (2015).

[35] J. R. Wang, X. D. Chen, F. L. Zhao, and J. W. Dong, Full polarization conical dispersion and zero-refractive-index in twodimensional photonic hypercrystals, Sci. Rep. 6, 22739 (2016).

[36] S. Kita, Y. Li, P. Camayd-Munoz, O. Reshef, D. I. Vulis, R. W. Day, E. Mazur, and M. Loncar, On-chip all-dielectric fabrication-tolerant zero-index metamaterials, Opt. Express 25, 8326 (2017).

[37] H. Guo, H. Liu, X. Zhang, H. Chen, W. Liu, S. Wang, and Y. Cui, Dirac point and cloaking based on honeycomb lattice photonic crystal, Appl. Phys. Express, 6, 042003 (2013).

[38] X. T. He, Z. Z. Huang, M. L. Chang, S. Z. Xu, F. L. Zhao, S. Z. Deng, J. C. She, and J. W. Dong, Realization of zero-refractiveindex lens with ultralow spherical aberration, ACS Photonics 3, 2262 (2016).

[39] X. T. He, Y. N. Zhong, Y. Zhou, Z. C. Zhong, and J. W. Dong, Dirac directional emission in anisotropic zero refractive index photonic crystals, Sci. Rep. 5, 13085 (2015).

[40] A. Y. Piggott, J. Lu, K. G. Lagoudakis, J. Petykiewicz, T. M. Babinec, and J. Vuckovic, Inverse design and demonstration of a compact and broadband on-chip wavelength demultiplexer, Nat. Photon. 9, 374 (2015). 
[41] B. Shen, P. Wang, R. Polson, and R. Menon, An integratednanophotonics polarization beamsplitter with $2.4 \times 2.4 \mu \mathrm{m}^{2}$ footprint, Nat. Photon. 9, 378 (2015).

[42] E. Bor, M. Turduev, and H. Kurt, Differential evolution algorithm based photonic structure design: Numerical and experimental verification of subwavelength $\lambda / 5$ focusing of light, Sci. Rep. 6, 30871 (2016).

[43] L. H. Frandsen, Y. Elesin, L. F. Frellsen, M. Mitrovic, Y. Ding, O. Sigmund, and K. Yvind, Topology optimized mode conversion in a photonic crystal waveguide fabricated in silicon-oninsulator material, Opt. Express 22, 8525 (2014).

[44] J. M. Geremia, J. Williams, and H. Mabuchi, Inverse-problem approach to designing photonic crystals for cavity QED experiments, Phys. Rev. E 66, 066606 (2002).

[45] L. Shen, Z. Ye, and S. He, Design of two-dimensional photonic crystals with large absolute band gaps using a genetic algorithm, Phys. Rev. B 68, 035109 (2003).

[46] O. Sigmund, and K. Hougaard, Geometric Properties of Optimal Photonic Crystals, Phys. Rev. Lett. 100, 153904 (2008).

[47] M. Turduev, E. Bor, C. Latifoglu, I. H. Giden, Y. S. Hanay, and $\mathrm{H}$. Kurt, Ultra-compact photonic structure design for strong light confinement and coupling into nano-waveguide, J. Light. Technol. 36, 2812 (2018).
[48] S. Johnson and J. D. Joannopoulos, Block-iterative frequencydomain methods for Maxwell's equations in a planewave basis, Opt. Express 8, 173 (2001).

[49] K. V. Price, R. M. Storn, and J. A. Lampinen, Differential Evolution: A Practical Approach to Global Optimization (SpringerVerlag, New York, 2005).

[50] J. Berenger, A perfectly matched layer for absorption of electromagnetic waves, J. Comput. Phys. 114, 185 (1994).

[51] A. F. Oskooi, D. Roundy, M. Ibanescu, P. Bermel, J. D. Joannopoulos, and S. G. Johnson, Meep: A flexible free-software package for electromagnetic simulations by the FDTD method, Comput. Phys. Commun. 181, 687 (2010).

[52] M. Mutlu, A. E. Akosman, A. E. Serebryannikov, and E. Ozbay, Diodelike Asymmetric Transmission of Linearly Polarized Waves Using Magnetoelectric Coupling and Electromagnetic Wave Tunneling, Phys. Rev. Lett. 108, 213905 (2012).

[53] C. Wang, C. Zhou, and Z. Li, On-chip optical diode based on silicon photonic crystal heterojunctions, Opt. Express 19, 26948 (2011).

[54] B. Shen, R. Polson, and R. Menon, Integrated digital metamaterials enables ultra-compact optical diodes, Opt. Express 23, 10847 (2015). 